\title{
MAGNETIC TREATMENT OF LIQUID FOR POTATO LOSS REDUCTION AT STORAGE
}

\author{
Alexander Lysakov, Gennady Nikitenko, Anton Kryukov, Yaroslav Tarasov \\ Stavropol State Agrarian University, Russia \\ s_lysakov@mail.ru, nikitenko_gv@mail.ru,kryukovar@energomera.ru, tarasov-yar@mail.ru
}

\begin{abstract}
For seed and table potatoe storage a proper relative humidity level of at least $95 \%$ should be maintained. The decreased relative humidity of less than $95 \%$ causes the increase in potato tubers' weight loss up to $40 \ldots 50 \%$. Specific devices called humidifiers are used to humidify the air in potato-storage houses. There are two dominant types of humidifiers that throw water in form of vapor or small-drop suspension (aerosol). The humidifiers of the second type do their primary task and also reduce the temperature, which is a great advantage. Humidifiers operate with a large liquid flow rate. Techniques used to reduce the liquid flow rate and increase the relative humidity level in a potato-storage house include magnetic water treatment. Pilot studies have found that the consumption of liquid depends on the parameter called a treatment dose. The parameter is a product of magnetic induction by treatment time $(\mathrm{mT} \cdot \mathrm{s})$. Pilot studies have found that for the treatment dose within $10 \ldots 12 \mathrm{mT} \cdot \mathrm{s}$, a liquid flow consumption for air humidification decreases by $60 \ldots 70 \%$, potato losses are less than $5 \%$ after 150 days of storage. Additionally, the research was conducted with the storage temperature from $4 \ldots 6^{\circ} \mathrm{C}$ to $12 \ldots 14{ }^{\circ} \mathrm{C}$. In this case, for the treatment dose within $10 \ldots 12 \mathrm{mT} \cdot \mathrm{s}$, liquid flow consumption for air humidification decreases by $50 \ldots 60 \%$, potato losses are less than $8 \%$ after 150 days of storage. As a result of experiments, the design of a new device for magnetic processing of liquids has been developed. It is anticipated that magnetic treatment of liquids influences the potato cells, as the result of the effects, potato tubers either lock in moisture, starch and other substances, or lose them quickly due to inner-structure destruction.
\end{abstract}

Keywords: weight, potato, magnet, loss.

\section{Introduction}

The main purpose of potato storage is to keep the highest quality crop with minimum losses for a long period, which can be up to 8 to 9 months.

To ensure long-term potato storage, temperature, humidity, and the presence of oxygen are of great importance, and, in the absence of light, they are regulated with ventilation.

Ventilation is vital for proper potato storage. In an oxygen-free environment, the tubers lack respiration and die. If oxygen is not enough, the tubers' pulp turns black, especially in the core. Change in ventilation can regulate all the factors affecting storage: temperature, humidity, and air exchange [1].

The healing period that starts immediately after harvesting, often referred to as the wound healing period, is one of the vital stages of storage. The maximum weight losses occur during the healing period because of loss of moisture through the cuts and impact injuries and, also, high respiration rates. During the first month, from two to four percent of tubers weight are lost with evaporation. With proper storage, the evaporating moisture can be used to maintain the high relative humidity required during the wound healing period [2].

The wound healing period duration is 40-45 days, the optimum temperature at this period is 18 $19{ }^{\circ} \mathrm{C}$, humidity is $90-95 \%$. For the healing period, potatoes can be placed in a temporary storage. During the healing period, the ware potato should be in the darkroom, and the seed potato can be exposed to light and turn green. At the end of the healing period, all latent lesions in tubers become visible, therefore, before placing them for permanent storage, all tubers are examined, and the damaged ones are removed. For example, if the relative humidity is low or the potato is not dry enough, a starch layer can generate on the tuber cut and prevent healing [3].

Long-term storage of ware potato requires a low temperature of $2-3{ }^{\circ} \mathrm{C}$ to prevent sprouting. The average humidity during long-term storage is between 85 and $95 \%$. At higher humidity, early sprouting occurs. At moisture levels below 70\%, the potato loses firmness [4-5].

Using ventilation in large potato storage facilities requires applying numerous temperature and humidity sensors, fans, cooling and ventilation systems and, as a result, high electricity consumption. Therefore, all storage owners strive to reduce their electricity costs.

Currently, research is conducted to find out the effect of electric and magnetic fields on reducing potato weight loss; research on the use of vacuum packages and gas packages for storing potato, research on the gamma radiation effect on potato weight loss and amount of sugar, starch, and vitamins in tubers 
[6-8]. Also, a research is conducted on the biodynamic drug and chemical inhibitor effect on the potato tubers' quality during long-term storage.

There are a large number of experimental studies results on the use of magnetic water treatment to reduce the scale buildup on the pipes and heating boiler surfaces, on the use of magnetic water treatment for watering plant seeds to increase yields, and many other methods using magnetic water treatment in agriculture and industry $[9 ; 10]$. However, there are no studies to determine the effect of magnetic water treatment on potato preservation.

\section{Materials and methods}

The experimental investigation objective was to study the effect of magnetic water treatment used in the humidification system on the change in potato weight loss.

During the experiment, the following research methods were used: the method of multifactor experiment, statistical analysis, determination of data adequacy [11].

The experiment and mathematical processing of the experimental data were conducted according to the following plan:

1. Deciding on equipment, the number of experiments, expenses;

2. Analysis of measurement accuracy;

3. Adjustment and calibration of devices, verification of manufactured samples (gaps, tolerances, etc.);

4. Measurement quality check;

5. Finding out the largest measurement error and rejection of repeated measurements;

6. Conversion of the measured parameters from absolute values to relative values;

7. Finding the deviation of the parameters of the original from the parameters of the model;

8. Finding the variance and standard deviation;

9. Data smoothing in tables and graphs;

10. Interpolation and extrapolation of functional dependencies.

For the experiment, a model of storage chamber was designed consisting of several isolated sections. Every section contained $30 \mathrm{~kg}$ potatoes of the Aurora variety. Temperature and humidity sensors were placed in every section. The temperature range was $4-6^{\circ} \mathrm{C}$, the humidity range was 92 $95 \%$. The potato mass was measured after $30,60,90,120,150$ days of storage.

An ultrasonic air humidification system of the TM - Nova brand was used as a device for creating high humidity, since it is commonly available in the southern regions of the Russian Federation. The ultrasonic air humidification system splits water into a stable tiny water aerosol (fog) with a diameter of water particles 1-5 microns with a specific ultrasonic method using ceramic discs. Forced by the blower fan, the generated water mist moved from the exhaust duct of the humidifier into the room and having mixed with the air in the chamber evaporates with increasing humidity. Water aerosol of a given dispersion (1-5 microns) creates a mixture with the air and spreads through the chamber with the air flows and also with the airflow produced by the cooler's fan.

As part of the air humidification system, there is a tank for the liquid that later turns into fog. In the course of the experiment, the liquid undergone electromagnetic treatment ran to the tank. A universal apparatus for magnetic substance treatment, invented by the authors of the article, was used as an apparatus for electromagnetic water treatment.

This apparatus for electromagnetic water treatment is fundamentally new, allowing improve both the process of magnetic treatment and its implementation means. The design of the apparatus and manufacturing technology allow adjust it for a specific task of magnetic substance treatment and use devices of various efficiency and with different diameters of the passage section. The apparatus is of very simple design, since it includes body 1 , coil frame 2 , besides, between the two halves of body 1 , rubber seals 4 and 5 are provided to make magnetizing coil 3 moisture-resistant (Figure 1).

To install the apparatus on the pipeline, flanges 6 were welded to the outer parts of the body 1 . The frame of the magnetizing coil, in its axial section, has thin walls alternating with thickenings.

The scheme of the apparatus shows a thin wall $(\delta)$ with poles located on its sides. The magnetic field character largely depends on the length of the thin wall and the pole offset angle. A change of one 
of these parameters always results in a change in the magnetic field, since the resistance of the magnet gap in both air and steel also changes. It is expected to obtain a magnetic field in the treatment area by changing the passing magnetic flux cross-section, which will result in ferromagnetic material saturation in places with minimal cross-section. Due to saturation in this area, the main magnetic flux will be pushed into the surrounding space. To increase or decrease the magnetic induction value in the treatment area, it is necessary to change the length of the thin wall.

The apparatus operates as follows. When an alternating or direct current is supplied to the magnetizing coil 3, a magnetic flux $\Phi$ is formed (Figure 1) with its lines of force closed along the body 1 and the frame 2 . The thin wall of the frame 2 operates in saturation mode and provides a significant magnetic resistance to the flux $\Phi$, which is divided into flows: $\Phi_{1}$ - is a magnetic flux in the volume of the magnetizing coil; $\Phi_{2}$ - is a magnetic flux closing along the thin wall; $\Phi_{3}-$ is a magnetic flux bulging towards the water under treatment.

The water flowing through the pipeline at a certain speed repeatedly crosses the lines of force of the magnetic flux $\Phi_{3}$ and is physically affected. In this apparatus for magnetic water treatment, the method of forming the magnetic workflux (by saturating the thin wall of the frame) is drastically changed. The apparatus unification made on the same body by replacing the frame 2 with the magnetizing coil 3 allows finding the optimal parameters of the magnetic field for any technological process.

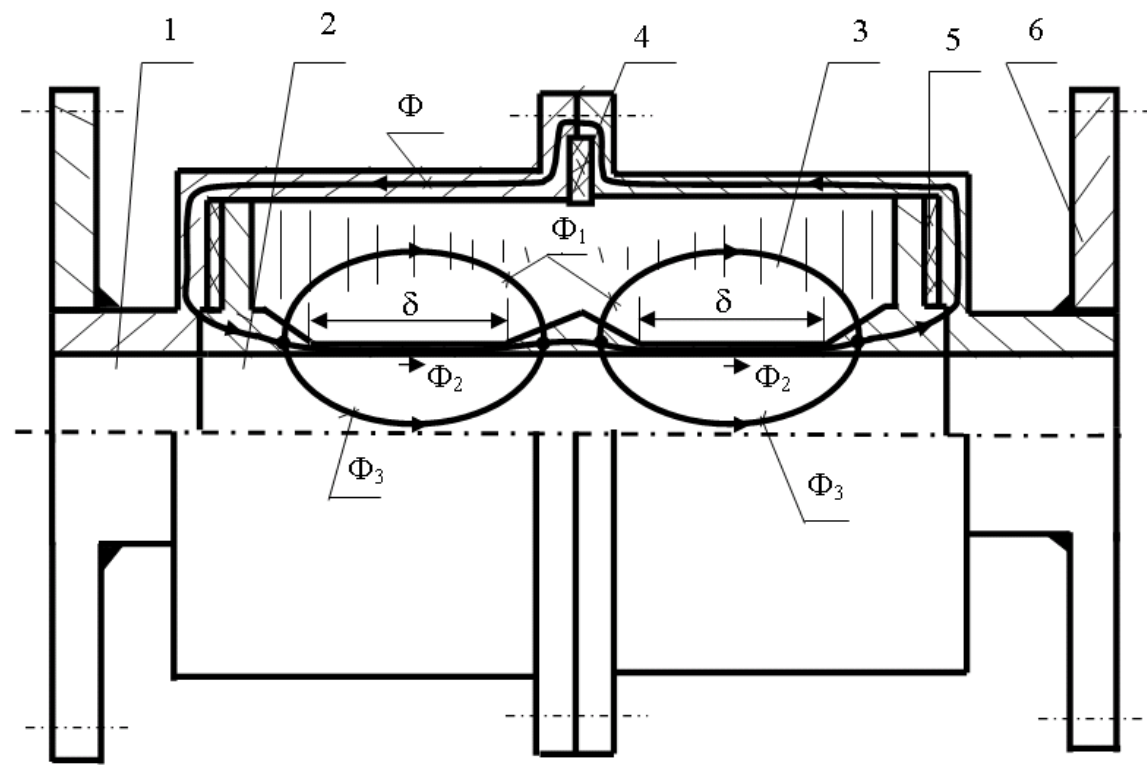

Fig. 1. Apparatus for electromagnetic water treatment: 1 - body;

2 - frame of the magnetizing coil; 3 - magnetizing coil; 4,5 - rubber seals; 6 -flange

Electromagnetic water treatment was with various combinations of treatment time and electromagnetic induction. The electromagnetic treatment parameters are as follows: $10 \pm 2 \mathrm{mT} \cdot \mathrm{s}$, $20 \pm 2 \mathrm{mT} \cdot \mathrm{s}, 30 \pm 2 \mathrm{mT} \cdot \mathrm{s}, 40 \pm 2 \mathrm{mT} \cdot \mathrm{s}, 50 \pm 2 \mathrm{mT} \cdot \mathrm{s}$.

\section{Results and discussion}

As a result of experimental studies, it was found that electromagnetic water treatment aimed at humidifying the air during potato storage can have both negative and positive consequences. With treatment parameters, $10 \pm 2 \mathrm{mT} \cdot \mathrm{s}$, the potato weight loss after 150 days was $5.2 \%$. All other experiment samples showed the value of potato weight loss above $5.2 \%$. The potato placed in the storage section with maintained humidity of $92-95 \%$ without electromagnetic water treatment lost $5.5 \%$ after 150 days of storage (Table 1).

Also, during the experiment, the length of the sprouts was measured, and the tuber surface colour was observed. The maximum sprout length of $10 \mathrm{~mm}$ was in the sample without electromagnetic treatment; on other samples, sprouts were absent or were less than $10 \mathrm{~mm}$. In all the experiment samples the tuber surface colour did not change. 
Effect of magnetic water treatment on potato preservation

Table 1

\begin{tabular}{|c|c|c|c|c|c|c|}
\hline \multirow{2}{*}{$\begin{array}{c}\text { Storage } \\
\text { time, } \\
\text { days }\end{array}$} & $\mathbf{1 0}$ & $\mathbf{2 0}$ & $\mathbf{3 0}$ & $\mathbf{4 0}$ & $\mathbf{5 0}$ & $\begin{array}{c}\text { Untreated } \\
\text { control }\end{array}$ \\
\cline { 2 - 7 } & \multicolumn{7}{|c|}{ Weight loss, \% } \\
\hline 30 & 2.2 & 3.5 & 3.6 & 4.1 & 4.5 & 3 \\
\hline 60 & 2.9 & 4 & 4.2 & 4.6 & 5 & 3.5 \\
\hline 90 & 3.5 & 4.8 & 4.5 & 5.4 & 5.8 & 4.2 \\
\hline 120 & 4.3 & 5.4 & 5.4 & 6 & 6.7 & 5.2 \\
\hline 150 & 5.2 & 5.7 & 6 & 6.4 & 7.2 & 5.5 \\
\hline
\end{tabular}

During the experiment, the rate of water flow necessary for fog formation in the storage section was also measured. In the section without electromagnetic treatment, water consumption to maintain humidity in the range of $92-95 \%$ was $2-2.5$ litres per hour. In the sections with electromagnetic treatment, the water consumption was 1.3-2.2 litres per hour. The minimum water consumption was in the samples with the electromagnetic treatment of $10 \pm 2 \mathrm{mT} \cdot \mathrm{s}$, which was up to 1.3-1.5 litres per hour. This method of reducing water consumption requires additional research.

In the course of the experiment, studies were carried out to increase the storage temperature from 4- $6^{\circ} \mathrm{C}$ to $12-14{ }^{\circ} \mathrm{C}$. The temperature increase was made only for two samples - without electromagnetic treatment and with electromagnetic treatment $10 \pm 2 \mathrm{mT} \cdot \mathrm{s}$. In the first sample, the weight loss was $9.4 \%$, in the second sample, the weight loss was $7.9 \%$. Water consumption in the humidification system was 2.5-3 litres per hour in the first sample, in the second sample $-2-2.5$ litres per hour.

\section{Conclusions}

As a result of the experimental studies, there are the following conclusions:

1. When storing potato in sections with the moisture content of $92-95 \%$, the weight loss after 150 days does not exceed $5.5 \%$;

2. With the electromagnetic treatment of water for a humidification system of $10 \pm 2 \mathrm{mT} \cdot \mathrm{s}$, the potato weight loss does not exceed $5.2 \%$ after 150 days of storage;

3. An increase in the storage temperature from $4-6^{\circ} \mathrm{C}$ to $12-14{ }^{\circ} \mathrm{C}$ leads to an increase in potato losses up to $9.4 \%$;

4. Electromagnetic treatment of water for the humidification system can reduce water consumption by $60-70 \%$ compared to the samples without electromagnetic treatment.

\section{References}

[1] Eltawil M., Samuel D., and Singhal O. Potato Storage Technology and Store Design Aspects. Agricultural Engineering International: the CIGR Ejournal. Invited Overview. No. 11. Vol. VIII. 2006.

[2] Mehta A., Singh B., Ezekiel R., Minhas J.S. Processing quality comparisons in potatoes stored under refrigerated and non-refrigerated conditions. Indian Journal of Plant Physiology, vol. 19 (2), 2014, pp. 149-155.

[3] Driskill Jr. E.P., Knowles L.O., Knowles N.R. Temperature-induced changes in potato processing quality during storage are modulated by tuber maturity. American Journal of Potato Research, vol. 84 (5), 2007, pp. 367-383.

[4] Paul V., Ezekiel R. Changes in temperature and relative humidity in heap and pit during storage of potatoes. Potato Journal, vol 32, 2005, pp. 205-206.

[5] Sijia Liu, Xiangning Chen, Jing Zhou. etc. Quality changes of different varieties of fresh-type potatoes during storage. Proceedings of International conference "IOP Conference Series: Materials Science and Engineering”, 562, 2019, 012123.

[6] Rezaee M., Almassi M., Minaei S. etc. Impact of post-harvest radiation treatment timing on shelf life and quality characteristics of potatoes. Journal of Food Science and Technology, vol. 50, 2013, pp. 339-345. 
[7] Starovoitov V., Manokhina A., Allayarov Zh. Dependence of tuber fluorescence on potato storage duration. Proceedings of International conference "IOP Conference Series: Earth and Environmental Science", 395, 2019, 012092.

[8] Lysakov A., Tarasov Y. Effect of magnetic field of neodymium ( $\mathrm{NdFeB}$ ) magnet on storage quality of potatoes. Proceedings of International conference "Engineering for Rural Development", 2019, pp. 609-612.

[9] Alzubaidy N. Effect of magnetic treatment of seeds and irrigation water at different intensities in the growth and production of maize. International Journal of Recent Scientific Research, vol. 5, issue 10, 2014 pp.1923-1925.

[10] Kozyrskyi V., Zablodskiy M., Savchenko V. etc. The Magnetic Treatment of Water Solutions and Seeds of Agricultural Crops. In book: Advanced Agro-Engineering Technologies for Rural Business Development, 2019, pp.256-292.

[11] Lysakov A., Nikitenko G., Konoplev E., Grinchenko V. Method to increase cleaning degree of electrostatic air cleaner. Proceedings of International conference "Engineering for Rural Development", 2018, pp. 554-559. 